\title{
A Retrospective Analysis of the Trajectory of Failed Back Surgery Syndrome Patients between their Last Spine Surgery and Spinal Cord Stimulation
}

Erik Van de Kelft, $M D, P h D^{1^{*}}$, Jean-Pierre Van Buyten, $M D, P h D^{2}$, Noemie Thomas, $M S c, M B A, M B B^{3}$, Surajit Basu, MD', Nasir Quraishi, LLM, FRCS ${ }^{5}$

${ }^{1}$ Department of Neurosurgery, Gen. Hosp. Nikolaas, Sint-Niklaas, Faculty of Medicine and Health Sciences, Antwerp University, Antwerp, Belgium

${ }^{2}$ Multidisciplinary Pain Centre, Gen. Hosp. Nikolaas, Sint-Niklaas, Belgium

${ }^{3}$ Department of Integrated Health Solutions, Medtronic Trading International SARL, Switzerland

${ }^{4}$ Department of Neurosurgery, Queens Medical Centre, Nottingham, UK

${ }^{5}$ Centre for Spine Studies \& Surgery, Queens Medical Centre, Nottingham, UK

\begin{abstract}
Spinal surgery for patients with degenerative lumbar spine disorder has a high success rate, but some patients experience postoperative back and leg pain (POBLP). Some of these patients are diagnosed to have Failed Back Surgery Syndrome (FBSS). Spinal cord stimulation (SCS) has been documented to be an effective treatment for FBSS. The success rate of SCS is higher in patients who are treated within one year after their last spine surgery. This retrospective analysis of hospital records of patients receiving SCS for failed back surgery syndrome (FBSS) aims at evaluating the current trajectory FBSS patients follow and to record the time delay between their last spine surgery and SCS trial.

The records of patients from two tertiary referral centers who received SCS for FBSS between 2011 and 2014 were retrieved. The time between last spine surgery and SCS trial was calculated and the number of events (outpatient visits, diagnostic imaging procedures and interventional pain therapy) in this time lapse were counted.

The mean time between the last spine surgery till trial SCS is 41 months (SD 18 months) for Queen's Medical Center and 31 months (SD 24 months) for AZ Nikolaas Hospital. In this time period, the patients had a mean of 4.5 outpatients visits, 11 diagnostic imaging procedures and 3 interventional pain therapies.

In patients suffering from FBSS, the long time delay between last spine surgery and trial SCS, and the high number of events patients are exposed to during this period, indicate potential for pathway improvement.
\end{abstract}

\section{Keywords}

Spine surgery, Degenerative spine disease, Postoperative back and leg pain, Failed back surgery syndrome, Spinal cord stimulation, Lead time, Multidisciplinary pain management, Referral process

\section{Introduction}

Chronic low back and leg pain (CBLP) related to the degenerative lumbar spine is one of the most prevalent nonfatal conditions worldwide and a significant cause of longterm disability [1-5]. Although most of these patients receive a conservative care program, an increasing number is offered spine surgery [5]. In most of these cases, spine surgery is offered to deal with nociceptive pain due to neuronal compression and/or instability of a lumbar segment. The spine surgery success rate can be as high as $89 \%$ (depending on the type of spine surgery) [6] but there is a percentage of patients that experiences persistent postoperative back
*Corresponding author: Dr. Erik Van de Kelft, Department of Neurosurgery, Gen Hosp Nikolaas, Moerlandstraat, 1, B-9100 Sint-Niklaas, Faculty of Medicine and Health Sciences, Antwerp University, Antwerp, Belgium, Tel: +32-3760-21-72, Fax: +323760-20-55

Accepted: March 20, 2019

Published online: March 22, 2019

Citation: Van de Kelft E, Van Buyten JP, Thomas N, et al. (2019) A Retrospective Analysis of the Trajectory of Failed Back Surgery Syndrome Patients between their Last Spine Surgery and Spinal Cord Stimulation. J Neurosurg Res Rev 2(1):22-26 
Citation: Van de Kelft E, Van Buyten JP, Thomas N, et al. (2019) A Retrospective Analysis of the Trajectory of Failed Back Surgery Syndrome Patients between their Last Spine Surgery and Spinal Cord Stimulation. J Neurosurg Res Rev 2(1):22-26

and leg pain (POBLP) [6]. In recent years, the phenotyping of POBLP has become increasingly important to guide the treatment selection after unsuccessfull spine surgery $[2-4,7-$ 9].

Repetitive surgical treatment in POBLP patients can only be an option, in case of mechanical nociceptive low back pain and/or nociceptive leg pain due to a persistent compression of a nerve root $[10,11]$. In some cases, the POBLP can be diagnosed as 'Failed Back Surgery Syndrome' (FBSS) [12]. FBSS is defined as "chronic radicular pain that has recurred or persists in the same distribution despite anatomically satisfactory previous surgery". It implies that previous surgery has not yielded the expected pain relief $[13,14]$. In FBSS, the POBLP is characterized by an incapacitating back and leg pain of a neuropathic type. If the postoperative pain consists mainly of neuropathic leg pain, the syndrome recently has been identified as Persistent Postoperative Neuropathic Pain (PPNP) [15]. In some POBLP patients, there is no indication for repetitive surgery nor a clear neuropathic pain pattern. These patients just do not reach the minimal clinically important difference (MCID) before and after spine surgery, for unknown reasons, although this MCID is seldom defined in daily practive nor in clinical studies [16].

For the FBSS/PPNP patients, spinal cord stimulation (SCS) has been documented to offer an effective treatment $[13,17$ 19]. A systematic review on the treatment options for FBSS shows that the strongest evidence for long-term treatment exists for SCS, with 1B evidence compared to conventional medical management and reoperation [20]. A study on the influence of SCS in FBSS patients with the generator either on or off showed a significant improvement in gait and posture when the generator was turned on [21]. Moreover, a case control study showed that the use of SCS in patients with FBSS was associated with reduced sick leave and disability pension [22]. Another retrospective analysis on the use of healthcare resources in over 120,000 FBSS patients with a follow-up of up to 9 years, showed improved long-term health economics to support the long-term cost utility of SCS [23-26]. The success rate of SCS for these two conditions is higher in patients who are treated within one year after their last unsuccessfull spine surgery [19]. Our clinical observation, however, learns that most patients follow a much longer trajectory of trial and error after their last spine surgery, before trial SCS. Due to a lack of a validated therapeutic algorithm or pathway for the management of POBLP patients, they frequently get lost between medical departments and/or undergo multiple diagnostic tests and therapeutic interventional pain treatments, prior to the diagnosis of FBSS/PPNP and prior to trial SCS.

We assume that the time delay in our tertiary referral centers between the last spine surgery and trial SCS is significantly more than one year and that this time delay is characterized by multiple and diverse diagnositic steps/ medical acts.

We retrospectively evaluated the trajectory and time delays between the last spine surgery and the trial for SCS in our FBSS/PPNP patients.

\section{Materials and Methods}

Two tertiary referral centers participated in this study: Queen's Medical Center (QMC) in Nottingham (United Kingdom) (centre 1) and AZ Nikolaas Hospital (AZN), in Sint-Niklaas (Belgium) (centre 2). Record cards of patients who received a trial SCS for FBSS/PPNP related to their last spine surgery, between 2011 and 2014 were retrieved. All patient information was anonymized and de-identified prior to analysis. The ethical committee of AZN gave approval to conduct this study (EC 17040).

The delay between the last unsuccessful spine surgery and trial SCS was registred in months. The number of 'events', defined as: Outpatient visits, interventional pain treatments and medical imaging, related to the POBLP were counted. The available data from centre 2 allowed separating interventional pain treatment procedures from outpatient visits, which could not be done for centre 1 .

A descriptive data analysis was performed using Excel. Outcome is given as mean, range and SD.

\section{Results}

In centre 1, 26 patients were identified as having a trial SCS for FBSS/PPNP. The mean time delay between the last unsuccessful spine surgery and trial SCS was 41 months (SD 18 months). In this time period, the patients underwent 16 (average 0.75 ) medical imaging procedures and patients had an average of 11 outpatient visits and/or interventional pain treatments related to their POBLP.

In centre 2, 41 patients who received a trial SCS for FBSS/ PPNP could be identified for this analysis. The mean time delay between the last unsuccessful spine surgery and trial SCS was 31 months (SD 24 months).

In this centre, the patient contacts with the hospital with respect to their POBLP ('events') could be split into medical imaging (diagnostic), outpatient visits (consultations) and interventional pain procedures (procedures). Between the last spine surgery and trial SCS, the mean number of events was 18 (SD 23). The mean number of consultations was 11 (SD 15), of medical imaging procedures 4.5 (SD 10) and of interventional pain procedures 3 (SD 4).

\section{Discussion}

This study confirms our assumption that in some cases of FBSS/PPNP in the POBLP patient, the time delay between last spine surgery and trial SCS is far more than one year; in fact it is 41 months in centre 1 and 31 months in centre 2 . The wide variation in time between the last spine surgery and the trial SCS for the FBSS/PPNP patient in our two referral centers, seems indicative for the absence of an established therapeutic algorithm for the POBLP patient. It might also take too long before the diagnosis of FBSS/PPNP in some POBLP patients is established, or, other less effective therapeutic trials are offered to the FBSS/PPNP patient for a long time period.

Despite being treated in tertiary SCS referral spine centers, FBSS/PPNP patients had not only a long time delay between their last spine surgery and trial SCS, they also had a high number of events prior to the trial SCS. 
Citation: Van de Kelft E, Van Buyten JP, Thomas N, et al. (2019) A Retrospective Analysis of the Trajectory of Failed Back Surgery Syndrome Patients between their Last Spine Surgery and Spinal Cord Stimulation. J Neurosurg Res Rev 2(1):22-26

To our knowledge, this is the first study that looked at these parameters in POBLP patients. As such, comparison of our results with the existing literature on this subject is impossible. Nevertheless, several attempts by different authors have been made to promote an algorithm or pathway for the management of POBLP patients $[27,28]$. In case of SCS, all authors are specifying the need for correct patient selection via a multidisciplinary team and are advocating early referral for trial SCS to improve treatment outcome $[27,28]$.

To make the time delay between the last unsuccessful spine surgery and trial SCS in case of FBSS/PPNP for POBLP patients shorter, three main issues have to be addressed; is there in the POBLP patient an indication for repeated spine surgery or is the postoperative pain characterized by a peripheral sensitization and can the diagnosis of FBSS/PPNP be confirmed, or is the pain within normal postoperative limits.

Repeated spine surgery can only offer decompression of nervous structure(s) and/or stabilization of evident instable segment(s), or corrections of complications such as pedicle screw malpositioning. Therefore the diagnostic process of patients with POBLP should first control for (residual, new) nerve compression and/or instability and should exclude complications. In the postoperative spine, compressed nervous structures can be identified by contrast enhanced Magnetic Resonance Imaging (MRI), whereas evident instability can be diagnosed by standing or dynamic X-Rays of the lumbar spine [29]. Major complications such as pedicle srews/cages, violating the spinal canal can be identified by these medical imaging procedures too. If no nervous structures are compressed and/or the POBLP is not due to an evident instability of the lumbar spine, and no complications necessitating additional surgery can be seen, no further spine surgery should be recommended.

The diagnosis of FBSS/PPNP can be made in case of a persistent incapacitating pain, characterized by a deafferentation pain in the back and/or the leg(s) while a persistent nociceptive pain due to neural comprerssion is excluded [12]. This takes two events (medical imaging). In this survey however, in centre 2 , a mean of 4.5 medical imaging procedures were carried out between the last spine surgery procedure and trial SCS but one patient even underwent 67 medical imaging procedures.

In centre 2, the mean number of events between the last surgery and trial SCS was 18 (range 2-112). This consisted of a mean of 11 outpatient visits, 4.5 imaging procedures and 3 interventional pain therapies. Although the mean figures seem reasonable, the wide range is indicative for the long trajectory some patients follow.

Some POBLP patients experience persistent pain, not due to persistent neuronal compression, a complication, or a mechanical instability and the diagnosis of FBSS/PPNP cannot be established. In most clinical practices a minimal clinically important difference (MCID) is not defined. As such, it is hard in this patient category to indicate if the postoperative result is within normal limits. Lack of sufficient pain alleviation after spine surgery may be encounterd, besides insufficient surgical decompression or FBSS. The POBLP patient should be evaluated for all possible facets of his bio-psycho-social conditions. But this is not the scope of this paper. We focused on the time delay and number of events in the FBSS/PPNP patient.

Once the diagnosis of FBSS is established, the best treatment option is SCS [30] preferentially with a trial that starts within the first year after the diagnosis [19].

Therefore, it is striking that the mean time delay between the last surgical procedure (which can be the first) and the trial SCS in centre 2 took 31 months, and in centre 1, 41 months. This clearly illustrates the absence of a generally accepted flow chart for POBLP patients or the reluctance to propose SCS, early after the FBSS/PPNP diagnosis. However, SCS has been proven the offer the best treatment option in case of FBSS.

With the actual medical knowledge of POBLP, FBSS and SCS, a simple managing and treatment flow-chart could be proposed (Figure 1). In each POBLP patient, one has to investigate whether nervous structures are still compressed and/or whether an evident spinal instability/complication is present. These are the three conditions in which repetitive

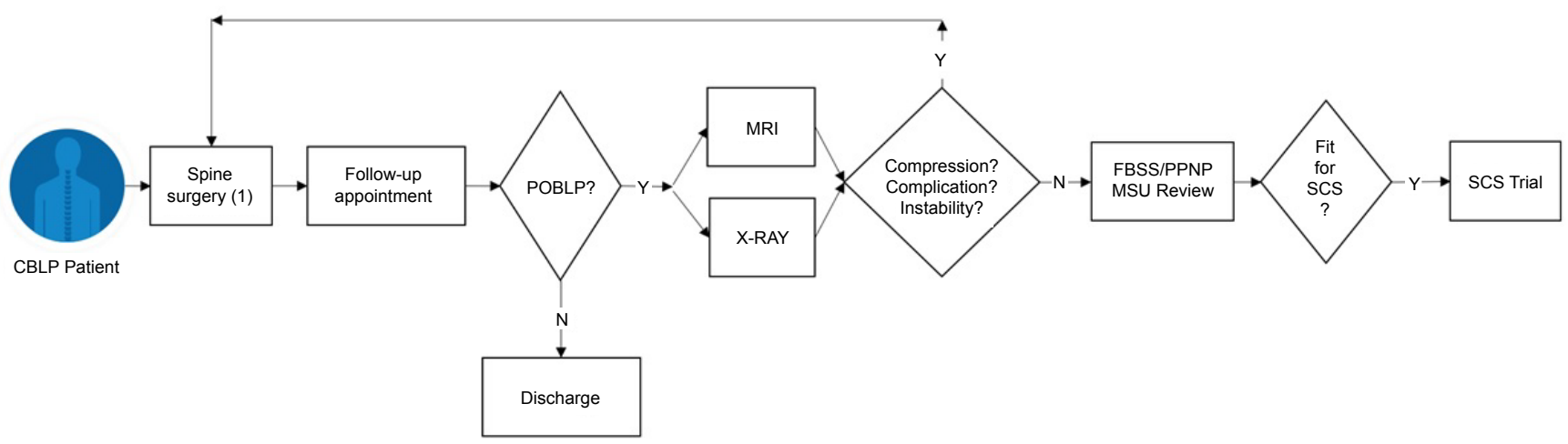

- Expected time from last Spine Surgery (1) to SCS Trial = less than 12 months

- Expected number of events from POBLP to SCS Trial = $\max 5$ (excluding SCS pathway)

Figure 1: Proposed high level failed back surgery syndrome patient flow. 
Citation: Van de Kelft E, Van Buyten JP, Thomas N, et al. (2019) A Retrospective Analysis of the Trajectory of Failed Back Surgery Syndrome Patients between their Last Spine Surgery and Spinal Cord Stimulation. J Neurosurg Res Rev 2(1):22-26

spine surgery can be considered. These conditions can be easily diagnosed by contrast enhanced MRI and dynamic $X$-Rays respectively. If these conditions do not exist, one should exclude the diagnosis of FBSS/PPNP. All conditions that can be dealth with by repeated spine surgery should be excluded and the pain must clearly have the characteristics of a peripheral sensitization. Existing questionnaires such as DN4 (Douleur Neuropathique en 4 questions) or LANSS (Leeds Assessment of Neuropathic Symptoms and Signs) could help spine surgeons to better identify the neuropathic nature of pain [13]. SCS has been documented to be the most effective treatment option for these patients, the sooner the better [27].

In our opinion there is no need for numerous other 'events' nor for a long time delay, once the diagnosis of FBSS/ PPNP is clear.

From this observation one might expect that an improved referral system, based on the previously described algorithm for POBLP patients, to a multidisciplinary spine unit (MSU) consisting of a team involving all the relevant specialists (neurosurgeon and/or orthopedic surgeon, anesthesiologistpain specialist, physical therapist, psychologist) may significantly reduce the time delay between the last spine surgery and the appropriate management of the medical problem. The importance of a MSU to assess these patients was described by Chan [31]. An early mutldisciplinary management should allow timely use of SCS, thus increasing the chances for success $[32,33]$.

The management of POBLP patients via a MSU is also expected to reduce the number of "events" the patients still undergo nowadays before obtaining trial SCS. This involvement of a MSU will most likely lead to an optimization of the hospital's resources and to a reduction of waste, limiting the time the patient spends to get the appropriate treatment and the number of 'events', embracing a more cost effective POBLP patient management process.

With emerging evidence and national policy guidance in some countries, it may be imperative now to adopt a pathway for early phenotyping of POBLP within the first 12 months of presentation and early trial of SCS.

The weakness of our study is the fact that the records were retrospectively retrieved from the hospital data-base, this means that there is not always information on the patient's trajectory outside the hospital, which can include surgery, diagnostic procedures and treatment in another hospital. Our findings, however, support the hypothesis that patients with POBLP follow a long trajectory that is a burden for the patient, his/her environment and society.

\section{Sources of Support}

This study was supported by an unrestricted grant and patient flow mapping/reporting in the two hospitals from Medtronic.

\section{References}

1. Hoy D, March L, Brooks P, et al. (2010) Measuring the global burden of low back pain. Best Pract Res Clin Rheumatol 24: 155-
165.

2. Vos $T$, Flaxman AD, Naghavi $M$, et al. (2012) Years lived with disability (YLDs) for 1160 sequelae of 289 diseases and injuries 1990-2010: A systematic analysis for the Global Burden of Disease Study 2010. Lancet 380: 2163-2196.

3. Sivasubramaniam V, Patel HC, Ozdemir BA, et al. (2015) Trends in hospital admissions and surgical procedures for degenerative lumbar spine disease in England: A 15-year time-series study. BMJ Open 5: e009011.

4. Kalff R, Ewald C, Waschke A, et al. (2013) Degenerative lumbar spinal stenosis in older people: Current treatment options. Dtsch Arztebl Int 110: 613-623.

5. Rajaee SS, Bae HW, Kanim LE, et al. (2012) Spinal fusion in the United States: Analysis of trends from 1998 to 2008. Spine (Phila Pa 1976) 37: 67-76.

6. Björn Strömqvist, Peter Fritzell, Olle Hägg, et al. (2014) SweSpine the Swedish spine register 2014 report.

7. Orita S, Yamashita T, Ohtori S, et al. (2016) Prevalence and location of neuropathic pain in lumbar spinal disorders: Analysis of 1804 consecutive patients with primary lower back pain. Spine (Phila Pa 1976) 41: 1224-1231.

8. Hoy D, March L, Brooks P, et al. (2014) The global burden of low back pain: Estimates from the Global Burden of Disease 2010 study. Ann Rheum Dis 73: 968-974.

9. Ragab A, Deshazo RD (2008) Management of back pain in patients with previous back surgery. Am J Med 121: 272-278.

10. Arts MP, Kols NI, Onderwater SM, et al. (2012) Clinical outcome of instrumented fusion for the treatment of failed back surgery syndrome: A case series of 100 patients. Acta Neurochir (Wien) 154: $1213-1217$.

11. Kim SS, Michelsen CB (1992) Revision surgery for failed back surgery syndrome. Spine (Phila Pa 1976) 17: 957-960.

12. Kumar K, Toth C (1998) The role of spinal cord stimulation in the treatment of chronic pain postlaminectomy. Curr Rev Pain 2: 8592.

13. Kumar K, Taylor RS, Jacques L, et al. (2007) Spinal cord stimulation versus conventional medical management for neuropathic pain: A multicentre randomised controlled trial in patients with failed back surgery syndrome. Pain 132: 179-188.

14. Thomson S (2013) Failed back surgery syndrome - definition, epidemiology and demographics. Br J Pain 7: 56-59.

15. Shamji MF, Shcharinsky A (2015) Use of neuropathic pain questionnaires in predicting persistent postoperative neuropathic pain following lumbar discectomy for radiculopathy. J Neurosurg Spine 24: 1-7.

16. Saltychev M, Eskola M (2018) Generalizing the results: How can we improve our reports? Eur Spine J 27: 977-979.

17. De La Porte C, Van de Kelft E (1993) Spinal cord stimulation in failed back surgery syndrome. Pain 52: 55-61.

18. Kumar K, Taylor RS, Jacques L, et al. (2008) The effects of spinal cord stimulation in neuropathic pain are sustained: A 24-month follow-up of the prospective randomized controlled multicenter trial of the effectiveness of spinal cord stimulation. Neurosurgery 63: 762-770.

19. Van Buyten JP (2016) Neurostimulation for the management of failed back surgery syndrome (FBSS). In: Surgery of the spine and 
Citation: Van de Kelft E, Van Buyten JP, Thomas N, et al. (2019) A Retrospective Analysis of the Trajectory of Failed Back Surgery Syndrome Patients between their Last Spine Surgery and Spinal Cord Stimulation. J Neurosurg Res Rev 2(1):22-26

spinal cord. A neurosurgical focus Van de Kelft E (edn), Berlin, Springer Verlag, 585-599.

20. Amirdelfan K, Webster L, Poree L, et al. (2017) Treatment options for failed back surgery syndrome patients with refractory chronic pain: An evidence based approach. Spine (Phila Pa 1976) 42: S41-S52.

21. Brugliera L, De Luca A, Corna S, et al. (2017) Spinal cord stimulation in failed back surgery syndrome: Effects on posture and gait-a preliminary 3D biomechanical study. Pain Res Manag 2017: 3059891

22. Kaijankoski H, Nissen M, Ikaheimo TM, et al. (2018) Effect of spinal cord stimulation on early disability pension in 198 failed back surgery syndrome patients: Case-control study. Neurosurgery.

23. Farber SH, Han JL, Elsamadicy AA, et al. (2017) Long-term cost utility of spinal cord stimulation in patients with failed back surgery syndrome. Pain Physician 20: E797-E805.

24. Waszak PM, Modric M, Paturej A, et al. (2016) Spinal cord stimulation in failed back surgery syndrome: Review of clinical use, quality of life and cost-effectiveness. Asian Spine J 10: 11951204.

25. Remacle T, Gilis N, Mauviel S, et al. (2018) Treating low back pain in failed back surgery patients with multicolumn-lead spinal cord stimulation. J Vis Exp 26.
26. Duy PQ, Anderson WS (2018) Two surgeries do not always make a right: Spinal cord stimulation for failed back surgery syndrome. Yale J Biol Med 91: 323-331.

27. Ganty P, Sharma M (2012) Failed back surgery syndrome: A suggested algorithm of care. Br J Pain 6: 153-161.

28. Pain SA, Pain SA, Raff M, et al. (2013) Spinal cord stimulation for the management of pain: Recommendations for best clinical practice. S Afr Med J 103: 423-430.

29. van de Kelft EJ, van Goethem JW, de La Porte C, et al. (1996) Early postoperative gadolinium-DTPA-enhanced MR imaging after successful lumbar discectomy. Br J Neurosurg 10: 41-49.

30. Taylor RJ, Taylor RS (2005) Spinal cord stimulation for failed back surgery syndrome: A decision-analytic model and cost-effectiveness analysis. Int J Technol Assess Health Care 21: 351-358.

31. Chan CW, Peng P (2011) Failed back surgery syndrome. Pain Med 12: 577-606.

32. Taylor RS, Desai MJ, Rigoard P, et al. (2014) Predictors of pain relief following spinal cord stimulation in chronic back and leg pain and failed back surgery syndrome: A systematic review and meta-regression analysis. Pain Pract 14: 489-505.

33. Kumar K, Rizvi S, Nguyen R, et al. (2014) Impact of wait times on spinal cord stimulation therapy outcomes. Pain Pract 14: 709-720. 